\title{
Strain Dependence of Dielectric Properties in Chlorinated Polyethylene Vulcanizate
}

\author{
Motosuke Naoki, Kozo Nakajima, Takuhei Nose, and Toshio Hata \\ Department of Polymer Chemistry, Tokyo Institute of Technology, \\ Ookayama, Meguro-ku, Tokyo, Japan.
}

(Received September 10, 1973)

\begin{abstract}
Complex dielectric constants as a function of elongational strain were measured over a frequency range of $10^{2}-3 \times 10^{5} \mathrm{~Hz}$ and a temperature range of $-65-40^{\circ} \mathrm{C}$ in chlorinated polyethylene vulcanizate. The temperature and extension dependences of the static dielectric constants were obtained. It was found that the dependence of the static dielectric constants on extension was negative in the vicinity of the glass transition region both in the liquid and glassy state, but this dependence seemed to vanish in the regions far above and below the glass transition temperature. Two dispersion processes, the so-called $\alpha$ and $\beta$ relaxation processes, were observed, and the temperature and extension dependences of those processes were obtained. Superpositions along temperature were possible both in the $\alpha$ and $\beta$ process. For the $\alpha$ process, the master curve superposed along temperature changed its shape with extension ratio, and the superpositions could not be made along elongational strain. For the $\beta$ process, the superposability along elongational strain could not be confirmed because of experimental uncertainties. Mobilities in molecular motions suggested by the apparent shift factors decreased with an increase in elongation in the $\alpha$ process, whereas they seemed to increase slightly in the $\beta$ process. The extension dependence of the glass transition temperature in dielectric measurements was obtained and compared with the results from other methods.
\end{abstract}

KEY WORDS Elongation / Chlorinated Polyethylene / Dielectric Constant / $\alpha$ Relaxation / $\beta$ Relaxation / Glass Transition / Activation Length /

There have been many publications on the dielectric properties of amorphous polymers under isotropic strain induced by applied pressure; e.g., for the $\alpha$ relaxation process by Williams, et al., ${ }^{1}$ and Saito, et al., ${ }^{2}$ and for the $\beta$ relaxation process by Koppelman, ${ }^{3}$ Williams, et al., ${ }^{4}$ and Saito, et al. ${ }^{5}$ The dependence of the $\alpha$ process on elongational strain has not been measured by dielectricity, but by use of sound velocity for some rubbers by Mason ${ }^{6}$ and for the present sample by the authors. ${ }^{7}$ However these experiments were not sufficient to discuss the molecular mobility and the superposability. In the present paper, we measure the dependence of the dielectric properties on elongational strain for amorphous chlorinated polyethylene vulcanizate, which has electrical dipole moments in the chain and no side groups, and we attempt to clarify some of the characteristics of molecular motions and mobilities in the vicinity of the glass transition region.

\section{EXPERIMENTAL}

\section{Material}

The chlorinated polyethylene sample for the present study is a commercial product of ShowaDenko Company, ${ }^{8}$ and contains $39.1 \mathrm{wt} \%$ of chlorine. Studies with X-ray and NMR by Saito $^{9,10}$ indicated that the present sample has no crystalline properties nor any side groups. The sequences of chlorine atoms on the polymer chains have been also studied, ${ }^{10}$ and it was found that chlorinated polyethylene had only a few sequences of polyvinylchloride. The vulcanization of this material was carried out by using dicumylperoxide at $150^{\circ} \mathrm{C}$ with no supplementary recipes. The test specimen was 
purified by repeating the swelling (by benzene, toluene and tetrahydrofuran) and extracting (by methanol and acetone) procedures. After drying up completely in a vacuum at $60^{\circ} \mathrm{C}$, a static electric load of about $10^{4} \mathrm{~V} / \mathrm{cm}$ was applied on the specimen at $60^{\circ} \mathrm{C}$ in a vacuum for two days in order to remove some of the ionic impurities. ${ }^{11}$ Before measurements the specimen was relaxed at $60^{\circ} \mathrm{C}$ for one day at an extension ratio of about 2.

Apparatus and Process of Dielectric Measurements

The measurements were made in a three terminal electrode system by using a transformer bridge (Type TR3B, Ando Electric Co.). Figure 1 shows an illustration of the apparatus. The test specimen, the electrodes, and a motor by which the specimen could be stretched at various lengths from outside were set in a chamber with a glass window. The chamber was immersed in a bath. The chamber was filled with dried nitrogen gas to avoid relaxations due to water and to prevent the specimen from oxidative degradation.

The test specimen was cut from a sheet $0.0988 \mathrm{~cm}$ thick into a shape $10 \mathrm{~cm}$ in length and $5 \mathrm{~cm}$ in width. Eight parallel lines were marked on the sample using a felt-pen; these lines were used, to measure the extension ratios with the aid of a traveling microscope. The extension ratio at $20^{\circ} \mathrm{C}$ was determined by averaging the eight lengths of these lines on various parts of the sample:

After the specimen had been clamped and stretched to the desired extension ratio, the temperature was raised to the highest temperatures at which the dielectric measurements were to be made. At this length and temperature, the stress produced was relaxed for one day before each measurement. Then, in the liquid state, the temperature was lowered succesively in an interval of about $7^{\circ} \mathrm{C}$, with the complex dielectric constants being measured after the specimen had been allowed to relax and reach a practically accessible thermodynamic equilibrium at each given temperature. In the glassy state, the measurements were performed under continuous cooling at a rate of about $0.1{ }^{\circ} \mathrm{C} / \mathrm{min}$. The complex dielectric constants were measured in the frequency range of $10^{2}-3 \times 10^{5} \mathrm{~Hz}$, the temperature range of $-65-40^{\circ} \mathrm{C}$ and the extension ratio range of $1-2$.

\section{Dilatometric Measurement}

For the unstretched specimen, the thermal expansivities for the liquid and glassy state and the glass transition temperature $T_{\mathrm{g}}$ were measured by dilatometry at a cooling rate of $0.3^{\circ} \mathrm{C} / \mathrm{min}$.

\section{RESULTS AND DISCUSSIONS}

\section{Thermal Expansivity and Correction Factors}

For the unstretched specimen, the values of the volume-temperature coefficient, $(\partial V / \partial T)_{P}$, were obtained as $6.31_{4} \times 10^{-4}$ and $2.26_{3} \times 10^{-4}$ $\mathrm{cm}^{3} / \mathrm{gK}$ in the liquid and glassy state respectively, at a cooling rate of $0.3^{\circ} \mathrm{C} / \mathrm{min}$. The dilatometric glass transition temperature, $T_{\mathrm{g}}$, was $-20.1^{\circ} \mathrm{C}$. Temperature effects on the dielectric constants because of the thickness of the sample were corrected using these values.

The extension correction factors for dielectric constants were calculated disregarding any volume change due to elongational strain. The value

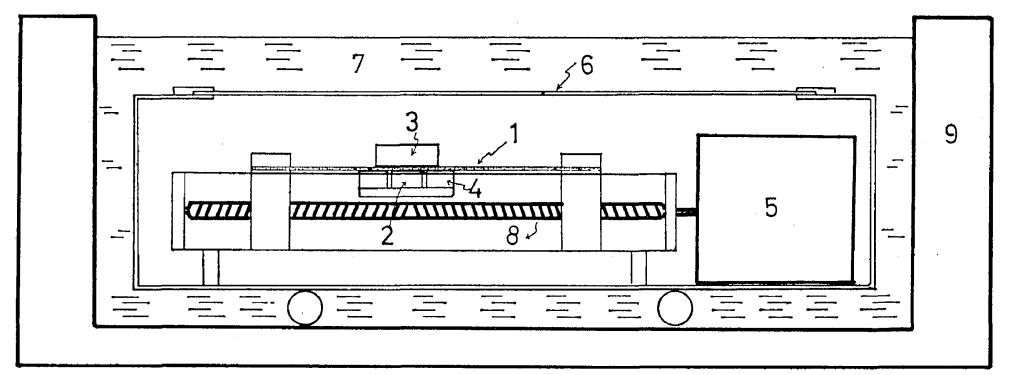

Figure 1. Apparatus for the measurement of the dielectricity: (1), sample; (2), main electrode; (3), sub electrode; (4), guard electrode; (5), motor; (6), glass window; (7), bath; (8), screw; (9), controller. 


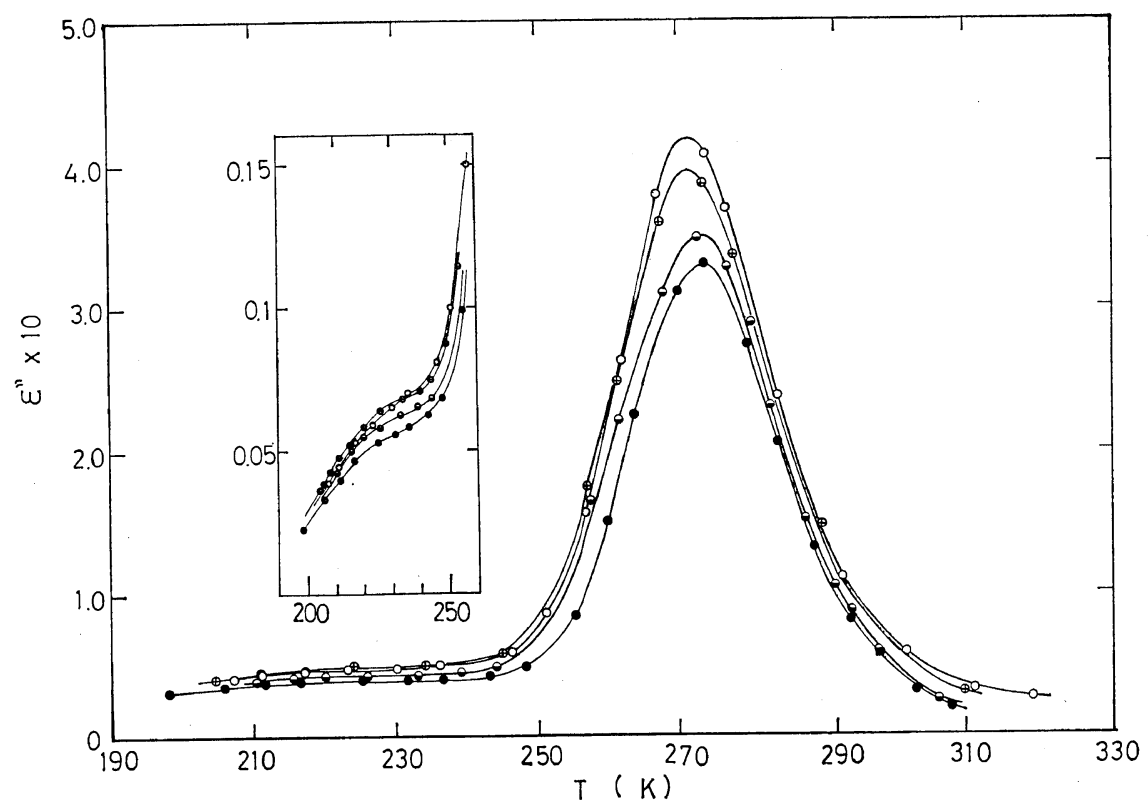

Figure 2. Variation of $\varepsilon^{\prime \prime}$ with temperature for constant length at $30 \mathrm{kHz}$. The extension ratios at $20^{\circ} \mathrm{C}$ were $\bigcirc, 1.00 ; \oplus, 1.22 ; \ominus, 1.56 ; \bullet, 1.95$.

of the volume-extension ratio coefficient, $(\partial \ln V / \partial \lambda)_{P T}$, was reported to be in the order of $10^{-4}-10^{-3}$ in the liquid region, ${ }^{12,13}$ and the change in volume with extension might be neglected in the liquid region. In the glassy region, however, the extension dependence of volume might be affected considerably by the glass forming conditions, i.e., cooling rates and extension ratios. Thus, the values of dielectric constants in the glassy region were the apparent ones, because of the lack of exact correction factors.

\section{Temperature Dependence of Dielectric Loss}

Figure 2 shows the plots of dielectric loss $\varepsilon^{\prime \prime}$ for several extension ratios as a function of temperature at $30 \mathrm{kHz}$. Pronounced loss peaks were observed at about $270^{\circ} \mathrm{K}$. These large peaks are the so-called $\alpha$ relaxation processes. It was found that the maximum value of $\varepsilon^{\prime \prime}$, $\varepsilon_{\max }^{\prime \prime}$, decreased with an increase in extension ratio, and the temperature $T_{\max }$ at which $\varepsilon^{\prime \prime}$ reaches $\varepsilon_{\max }^{\prime \prime}$ increased with increasing extension ratio. The $T_{\max }$ for the extension ratio of 1.95 was higher by about $2^{\circ} \mathrm{C}$ than that for the ratio of 1.00 .

So-called $\beta$ relaxation processes were observed in the range of $195-230^{\circ} \mathrm{K}$. The $\varepsilon_{\max }^{\prime \prime}$ for the $\beta$ relaxation process also decreased with an increase in extension ratio, but the dependence of $T_{\max }$ on extension was not clear in the plots of $\varepsilon^{\prime \prime} v s$. temperature.

\section{The $\beta$ Relaxation}

3-1. Static Dielectric Constants. Static dielectric constants in the glassy state, $\varepsilon_{20}$, and instantaneous ones, $\varepsilon_{\infty}$, were obtained from $\varepsilon^{\prime}-\varepsilon^{\prime \prime}$ plots.

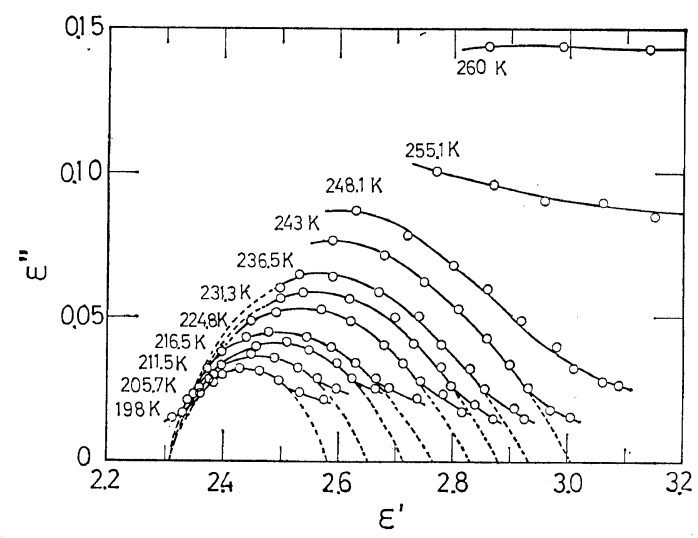

Figure 3. Complex plane plots in the $\beta$ process at the extension ratio of 1.95 . 


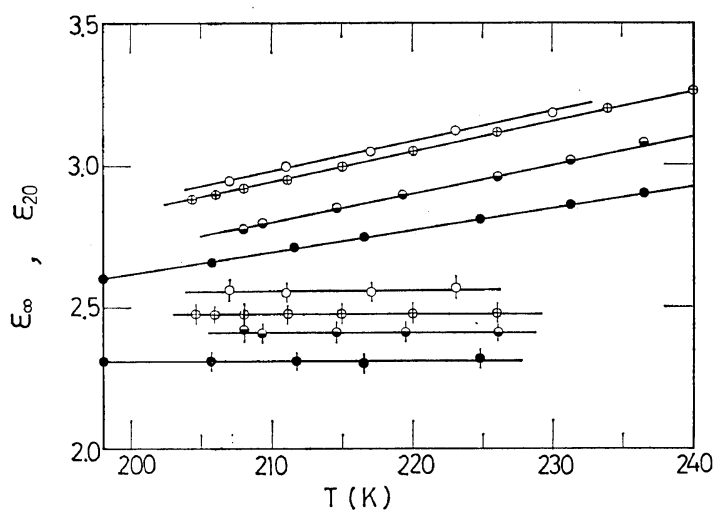

Figure 4. Variation of the static dielectric constant $O$ in the glassy state and the instantaneous one at constant length as a function of temperature. The extension ratios at $20^{\circ} \mathrm{C}$ were $\bigcirc, 1.00 ; \oplus, 1.22$; $\ominus, 156 ; \bullet, 1.95$.

An example of the $\varepsilon^{\prime}-\varepsilon^{\prime \prime}$ plots is shown in Figure 3. The temperature dependences of $\varepsilon_{20}$ and $\varepsilon_{\infty}$ are shown in Figure 4 for several extension ratios. The $\varepsilon_{\infty}$ depended only slightly on temperature but depended considerably on the extension ratio, as shown in Figure 3 and 4. The $\varepsilon_{20}$ changed linearly with temperature, and the slopes of these lines decreased with increasing

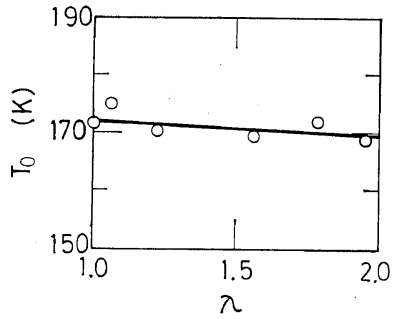

Figure 5. Variation of the apparent extinction temperature of the $\beta$ process with extension ratio.

extension ratio. The above relations on $\varepsilon_{20}$ and $\varepsilon_{\infty}$ are expressed by these empirical equations:

$$
\begin{gathered}
\varepsilon_{\infty}=2.53_{5}-0.26_{4}(\lambda-1) \\
\varepsilon_{20}=A_{20}\left(T-T_{0}\right)+\varepsilon_{\infty}
\end{gathered}
$$

where

$$
A_{20}=0.0112-0.0009(\lambda-1)
$$

and $T_{0}$ is the temperature at which $\varepsilon_{20}$ reaches $\varepsilon_{\infty}$. $\quad T_{0}$ as a function of extension ratio is shown in Figure 5. The apparent extinction point of the $\beta$ process, $T_{0}$, decreased slightly with an increase in extension ratio.

The relaxation intensity of the $\beta$ process, $\varepsilon_{20}-\varepsilon_{\infty}$, changed almost linearly with extension

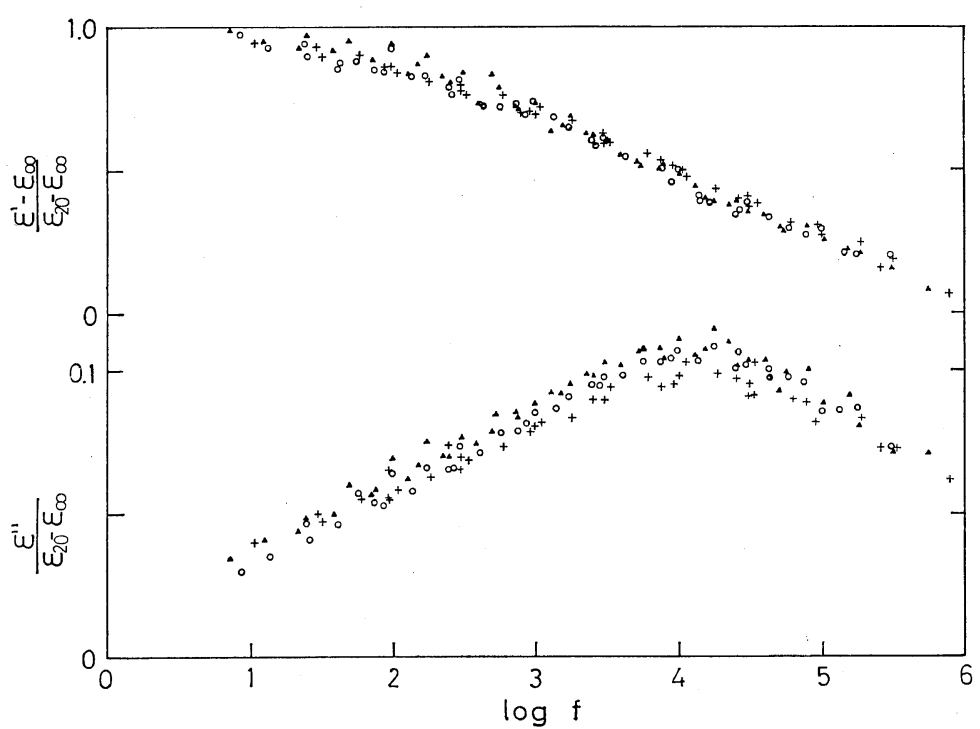

Figure 6. Master curves of $\left(\varepsilon^{\prime}-\varepsilon_{\infty}\right) /\left(\varepsilon_{20}-\varepsilon_{\infty}\right)$ and $\varepsilon^{\prime \prime} /\left(\varepsilon_{20}-\varepsilon_{\infty}\right)$ in the $\beta$ process as a function of frequency at extension ratios of $\boldsymbol{\Lambda}, 1.00 ;+, 1.45, \bigcirc, 1.95$. The reference temperature is $211.5^{\circ} \mathrm{K}$. 


\section{Strain Dependence of Dielectricity}

Table I. Static dielectric constants at the glass transition temperature

\begin{tabular}{ccccccccc}
\hline$T_{\mathbf{g}^{\mathrm{a}}},{ }^{\circ} \mathrm{K}$ & 253.1 & 253.4 & 253.7 & 254.1 & 254.2 & 254.6 & 254.7 \\
\hline$\lambda$ & 1.00 & 1.06 & 1.22 & 1.45 & 1.56 & 1.78 & 1.95 \\
$\varepsilon_{10}$ & 6.70 & 6.86 & 6.72 & 6.33 & 6.42 & 6.25 & 6.03 \\
$\varepsilon_{20}$ & 3.45 & 3.67 & 3.39 & 3.24 & 3.32 & 3.25 & 3.08 \\
$\Delta \varepsilon$ & 3.25 & 3.19 & 3.33 & 3.09 & 3.10 & 3.00 & 2.95 \\
$\varepsilon_{\infty}$ & 2.56 & 2.53 & 2.47 & 2.34 & 2.41 & 2.37 & 2.31
\end{tabular}

a Obtained as described in the text.

ratio, and the value of $\varepsilon_{20}-\varepsilon_{\infty}$ for every temperature decreased slightly with increasing extension ratio. The numerical results of the extrapolated values of $\varepsilon_{\infty}$ and $\varepsilon_{20}$ at $T_{\mathrm{g}}$ are listed in Table I.

3-2. Superpositions and Shift Factors. The frequency plots of $\left(\varepsilon^{\prime}-\varepsilon_{\infty}\right) /\left(\varepsilon_{20}-\varepsilon_{\infty}\right)$ and $\varepsilon^{\prime \prime} /\left(\varepsilon_{20}-\varepsilon_{\infty}\right)$ at temperatures sufficiently below $T_{\mathrm{g}}$ for the same extension ratios fell on a master curve within experimental errors. Thus superpositions along temperature were possible in the $\beta$ process. Figure 6 shows some examples of the master curves superposed along temperature, where the reference temperature is $211.5^{\circ} \mathrm{K}$. The value of $\varepsilon_{\max }^{\prime \prime} /\left(\varepsilon_{20}-\varepsilon_{\infty}\right)$ of the master curve for each extension ratio was different, but the shapes of the master curves for each extension

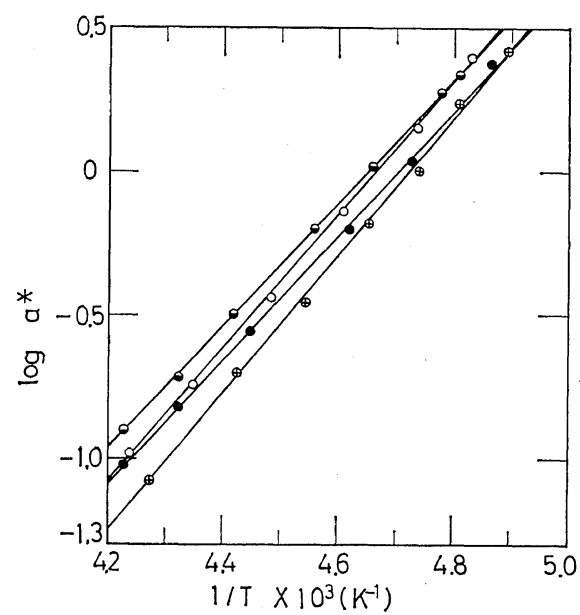

Figure 7. Variation of the apparent shift factor in the $\beta$ process at constant length as a function of reciprocal temperature. The extension ratios at $20^{\circ} \mathrm{C}$ were $\bigcirc, 1.00 ; \oplus, 1.22 ; \ominus, 1.56 ; \ominus, 1.95$.

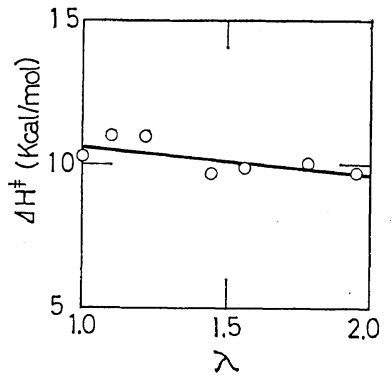

Figure 8. Variation of the activation enthalpy in the $\beta$ process with extension ratio.

ratio were rather similar.

Figure 7 shows the apparent shift factors $a^{*}$ against reciprocal temperature for several extension ratios. $a^{*}$ for the same extension ratio are the usual shift factors $a_{T}$ for the time temperature superposition, but the $a^{*}$ between different extension ratios are only apparent ones because the values of $a^{*}$ were obtained by shifting the master curves so that they had the same $f_{\text {max }}$. $a^{*}$ varied linearly with reciprocal temperature for each extension ratio. With respect to the extension dependence of $a^{*}$, noticeable uncertainties were observed and it was scarcely possible to see any pronounced dependences of $a^{*}$ on extension. However, at the lower temperatures, the $a^{*}$ decreased slightly with increasing extension ratio. In Figure 8, the activation enthalpy $\Delta H^{\neq}$is shown as a function of the extension ratio. The $\Delta H^{*}$ decreased slightly with increasing extension ratio.

3-3. Discussion. Observations with respect to the temperature dependence of the $\beta$ process may be summarized as follows:

(1) The static dielectric constant in the glassy state, $\varepsilon_{20}$, increased linearly with increasing temperature, as shown in Figure 4 and eq (3-2).

(2) Superpositions of the reduced values of $\varepsilon^{\prime}-\varepsilon_{\infty}$ and $\varepsilon^{\prime \prime}$ by $\varepsilon_{20}-\varepsilon_{\infty}$ along temperature were possible, as shown in Figure 6.

(3) The activation enthalpy $\Delta H^{\neq}$did not change with temperature in the region measured, as shown in Figure 7.

(4) The relaxation strength of the $\beta$ process seemed to vanish at a low temperature, as expressed by eq (3-2).

Observation (2) indicates that the mechanism 
and the distribution of relaxation times of the $\beta$ process might not change with temperature. The following observations with respect to the extension dependence of the $\beta$ process are given:

(5) $\varepsilon_{\infty}$ depended on extension ratio, as shown in Table $I$ and Figure 4.

(6) $\varepsilon_{20}-\varepsilon_{\infty}$ decreased with increasing extension ratio.

(7) Superpositions along extension ratio seemed not to be possible, as shown in Figure 6.

(8) $a^{*}$ decreased slightly with extension ratio.

(9) $\Delta H^{\neq}$decreased with extension ratio, as shown in Figure 8.

(10) $T_{0}$, at which $\varepsilon_{20}$ seemed to coincide with $\varepsilon_{\infty}$, decreased with increasing extension ratio.

The observed extension dependence of $\varepsilon_{\infty}$ exceeds the amount of the correction for density which is presumed by the extension dependence of the volume in styrene-butadiene copolymer in the glassy state. ${ }^{14}$ One reason for Observation (5) is thought to be that contact conditions of the test specimen with the electrodes might change slightly every time that the extension ratio was changed. This slight change in the contact conditions might be one of the causes of the fact that the experimental values as a function of extension ratio were all scattered, and, moreover, might be one of the obstructions in investigating the superposability of the $\beta$ process. There was nor essential difference between the shapes of the master curves for different extension ratios, as shown in Figure 6. If $\varepsilon^{\prime \prime} / \varepsilon_{\max }^{\prime \prime}$ is plotted against frequency, it is expected that the frequency plots at all extension ratios fall on the same curve within experimental errors.

The $\left(\varepsilon_{20}-\varepsilon_{\infty}\right) / \varepsilon_{\infty}$ ratio decreases with increasing extension ratio, similar to $\varepsilon_{20}-\varepsilon_{\infty}$ in Observation (6), in the temperature range measured. While Observation (10), moreover, indicates that $\left(\varepsilon_{20}-\varepsilon_{\infty}\right) / \varepsilon_{\infty}$ should increase with increasing extension ratio at least near $T_{0}$. These observations suggest that an inversion in the extension dependence of $\left(\varepsilon_{20}-\varepsilon_{\infty}\right) / \varepsilon_{\infty}$ may occur at about $190^{\circ} \mathrm{K}$. It is not clear whether this inversion has physical meaning or has merely arisen from the experimental uncertainties mentioned above.

Observations (8), (9), and (10) suggest that the stretch of the polymer chain facilitates the $\beta$ process, and the molecular mobility in the glassy state increases with increasing elongational strain. These observations may be due to the increase in the free volume with elongational strain. It is not clear, however, whether such a free volume concept is also able to explain the extension dependences of $\varepsilon_{20}-\varepsilon_{\infty}$ and $\left(\varepsilon_{20}-\varepsilon_{\infty}\right) / \varepsilon_{\infty}$.

\section{The $\alpha$ Relaxation}

4-1. Static Dielectric Constants. Static dielectric constants in the liquid state, $\varepsilon_{10}$, were obtained from $\varepsilon^{\prime}-\varepsilon^{\prime \prime}$ plots. Figure 9 shows the

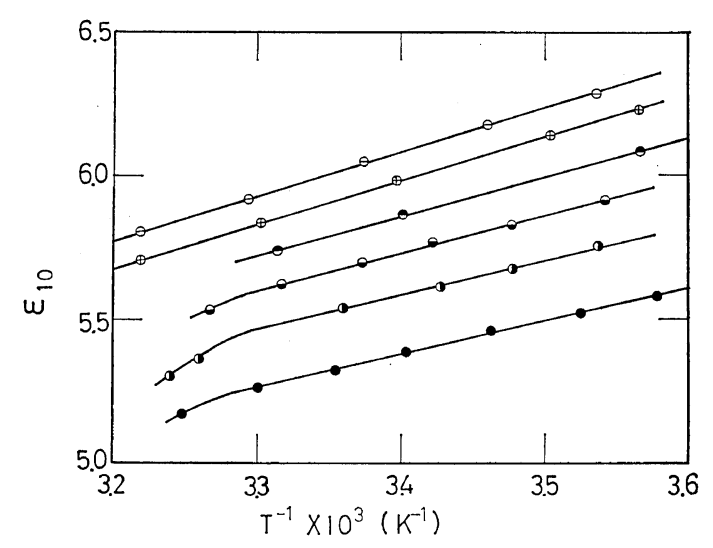

Figure 9. Variation of the static dielectric constant in the liquid state at constant length as a function of temperature. The extension ratios are at $20^{\circ} \mathrm{C}$ were $\ominus, 1.06 ; \oplus, 1.22 ; \ominus, 1.31 ; \mathcal{D}, 1.45 ; \bigcirc, 1.78$; , 1.95 .

plots of $\varepsilon_{10}$ as a function of reciprocal temperature for several extension ratios. It was found that $\varepsilon_{10}$ changed linearly with reciprocal temperature. In the high temperature region, the plots of $\varepsilon_{10}$ for the higher extension ratios deviated slightly downward from the linear line, and the curvature was opposite in sign to that estimated from the experimental equation of Ferry, et al. ${ }^{15}$ The empirical equation for the static dielectric constant in the liquid state, $\varepsilon_{10}$, is

$$
\varepsilon_{10}=A_{10} T^{-1}+B_{10}
$$

where

$$
\begin{aligned}
& A_{10}=1.39_{7}-0.17_{3}(\lambda-1) \times 10^{3} \\
& B_{10}=1.38_{6}-0.16_{0}(\lambda-1)
\end{aligned}
$$

The numerical extrapolated values of $\varepsilon_{10}$ at $T_{\mathrm{g}}$ 


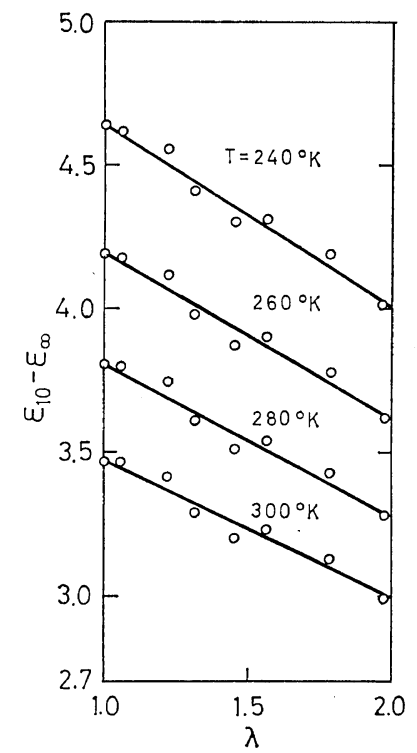

Figure 10. Variation of $\varepsilon_{10}-\varepsilon_{\infty}$ with extension ratio at constant temperature.

are shown in Table I. Figure 10 shows the dependence of $\varepsilon_{10}-\varepsilon_{\infty}$ on extension, where the $\varepsilon_{\infty}$ are the ones in the glassy state, which depended only slightly on temperature in the glassy state, as seen in Figure 4 . The value of $\varepsilon_{10}-\varepsilon_{\infty}$ for each temperature decreased considerably with an increase in extension ratio in a linear fashion, though noticeable uncertainties were observed.

4-2. Superpositions and Shift Factors. The frequency plots of $\varepsilon^{\prime \prime} / \varepsilon_{\max }^{\prime \prime}$ at all temperatures for the same extension ratio fell on a master curve in the liquid state. Thus the superpositions along temperature were possible in the $\alpha$ process. Figure 11 shows the master curves of $\varepsilon^{\prime \prime} / \varepsilon_{\max }^{\prime \prime}$ obtained by the superpositions along temperature for the extension ratio of 1.00 and 1.95 , where the reference temperature was $273.5^{\circ} \mathrm{K}$. The shape of the master curve depended on extension, i.e., the curve broadened with increasing extension ratio, and the superpositions along extension ratio were not possible in the liquid region, as shown in Figure 11.

Figure 12 shows the extension dependence of the apparent shift factors $a^{*}$. The values of $a^{*}$ between different extension ratios were obtained by the shifts of $f_{\max }$ of the master curves at $273.5^{\circ} \mathrm{K}$. $a^{*}$ increased with an increase in ex-

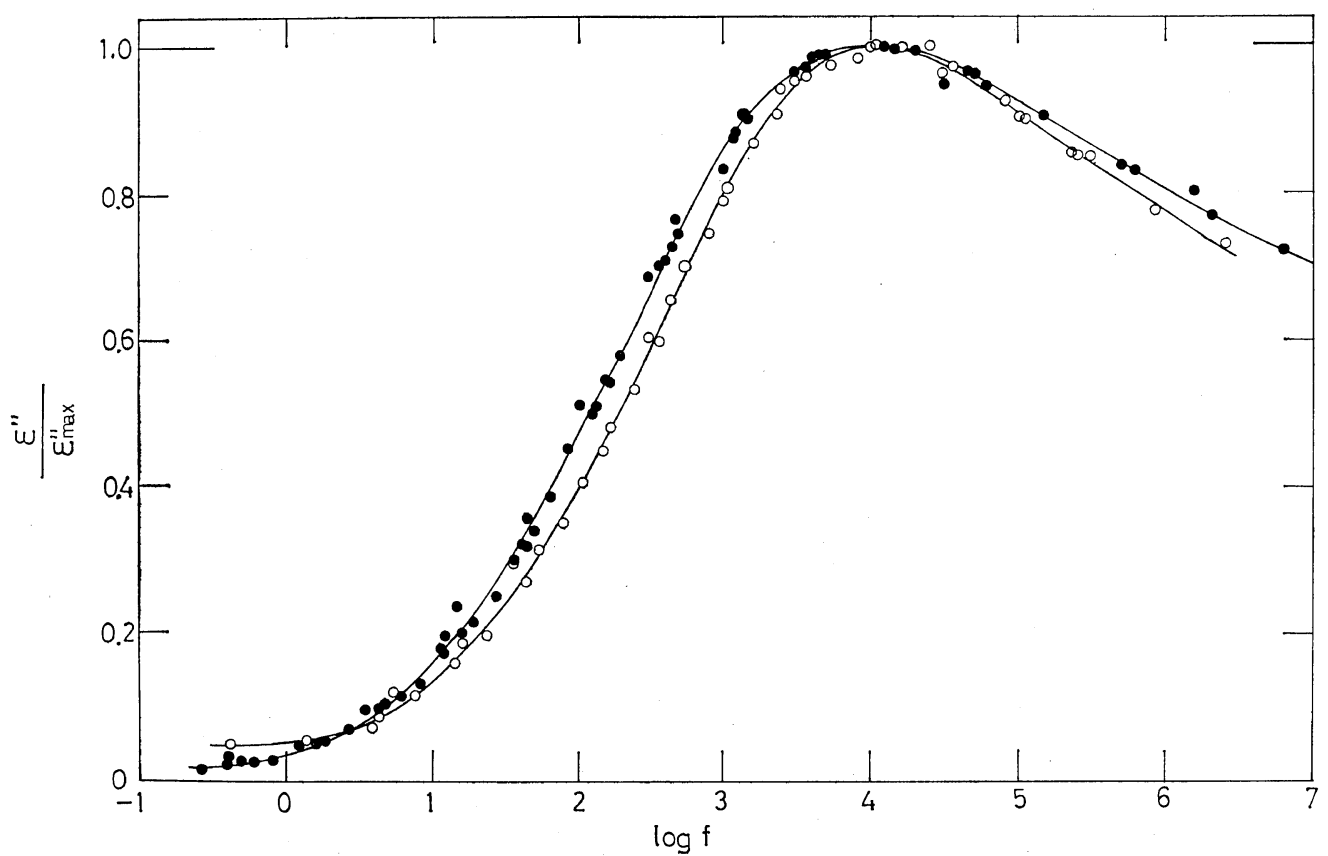

Figure 11. Master curves of $\varepsilon^{\prime \prime} / \varepsilon_{\text {max }}^{\prime \prime}$ in the $\alpha$ process as a function of frequency at extension ratios of $\bigcirc, 1.00$ and $0,1.95$. The reference temperature is $273.5^{\circ} \mathrm{K}$ 


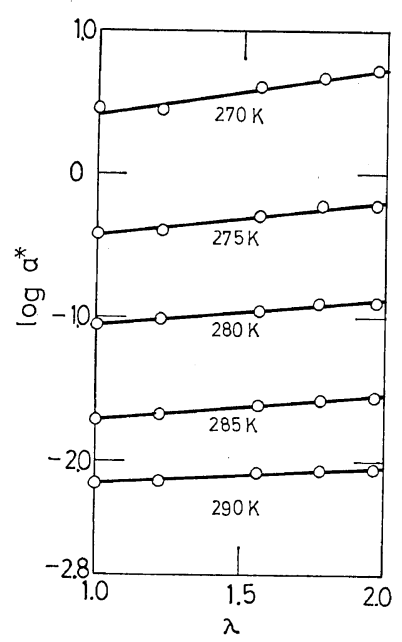

Figure 12. Variation of the apparent shift factor in the $\alpha$ process at constant temperature as a function of extension ratio.

tension ratio. The slope of the lines of $\log a^{*}$ $v s . \lambda$ (extension ratio at $20^{\circ} \mathrm{C}$ ) decreased with increasing temperature, as shown in Figure 13. In Figure 14, two examples of the temperature dependence of $\Delta H^{\neq}$are shown. The $\Delta H^{\neq}$ increased with increasing extension ratio over the entire temperature range above $T_{\mathrm{g}}$.

4-3. Discussion. The following observations with respect to the temperature dependences of

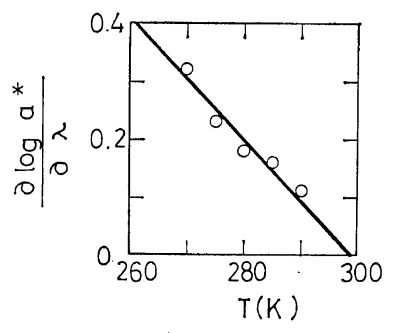

Figure 13. Variation of the magnitude of the strain dependence of the apparent shift factor in the $\alpha$ process as a function of temperature.

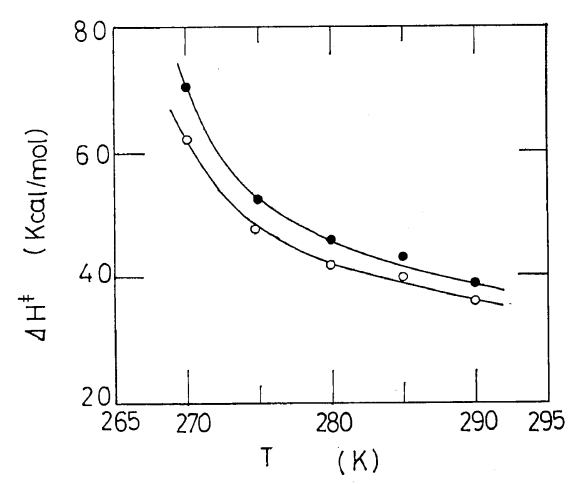

Figure 14. Variation of the activation enthalpy in the $\alpha$ process at constant length as a function of temperature. The extension ratios at $20^{\circ} \mathrm{C}$ were $\bigcirc, 1.00$ and $\bullet, 1.95$.

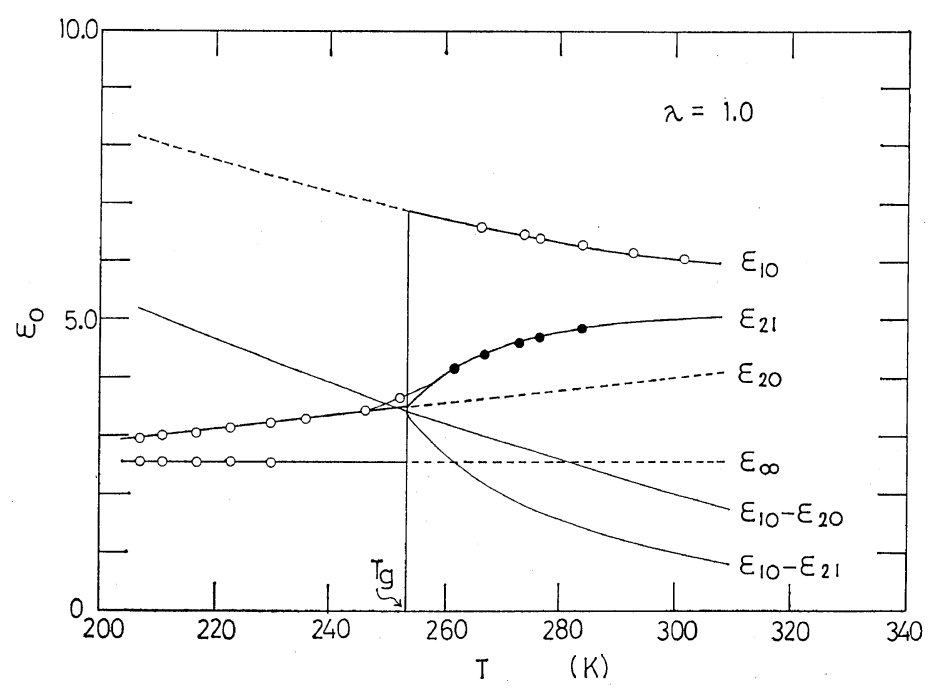

Figure 15. Variation of the static and instantaneous dielectric constants with temperature at the extension ratio of 1.00. The $\bigcirc$ and $\bigcirc$ indicate the experimental values and the calculated values as shown in the text, respectively. 
the $\alpha$ process in the liquid state can be made:

(11) The statistic dielectric constant in the liquid state, $\varepsilon_{10}$, increased linearly with increasing reciprocal temperature, as shown in Figure 9 and eq (4-1).

(12) Superpositions of the frequency plots of $\varepsilon^{\prime \prime} / \varepsilon_{\max }^{\prime \prime}$ along temperature were possible for the same extension ratio, as shown in Figure 11.

The relaxation strength of the $\alpha$ process is considered to be $\varepsilon_{10}-\varepsilon_{\infty}$ or $\varepsilon_{10}-\varepsilon_{20}$. If we regard the $\varepsilon_{\infty}$ in the glassy state to be identical with that in the liquid state, we obtain the value of $\varepsilon_{10}-\varepsilon_{\infty}$ for every temperature. However the frequency plots $\varepsilon^{\prime \prime} / \varepsilon_{10}-\varepsilon_{\infty}$ for every temperature do not fall on a curve. On the other hand, if we extrapolate the linear line of $\varepsilon_{20} v s$. temperature to the liquid region, we obtain the value of $\varepsilon_{20}$ for every temperature. However, the frequency plots of $\varepsilon^{\prime \prime} / \varepsilon_{10}-\varepsilon_{20}$ for each temperature also do not fall on the same curve. Therefore, superpositions along temperature cannot be applied to the $\alpha$ process in terms of $\varepsilon^{\prime \prime} / \varepsilon_{10}-\varepsilon_{\infty}$ and $\varepsilon^{\prime \prime} / \varepsilon_{10}-\varepsilon_{20}$. In other words, neither $\varepsilon_{10}-\varepsilon_{\infty}$ nor $\varepsilon_{10}-\varepsilon_{20}$ is competent to express the relaxation strength of the $\alpha$ process. If we assume the relaxation strength to be $\varepsilon_{10}-\varepsilon_{21}$, we can calculate the value of $\varepsilon_{21}$ from the temperature dependence of $\varepsilon_{\max }^{\prime \prime}$, because the superpositions of $\varepsilon^{\prime \prime} / \varepsilon_{\max }^{\prime \prime}$ were possible, as shown in Observation (11). In this case, the $\varepsilon_{21}$ no longer has a linear dependence on temperature, as shown in Figure 15 , which indicates a static dielectric constant map in the glass transition region at $\lambda=1$. A high frequency experiment is needed to verify the existence of such $\varepsilon_{21}$ in the liquid state.

The master curves of $\varepsilon^{\prime \prime} / \varepsilon_{\max }^{\prime \prime}$ in Figure 11 were broader than those of other typical amorphous polymers. One of reasons for this fact may be due to the wide distribution of the sequences of chlorine atoms. ${ }^{10}$

Observations with respect to the extension dependences of the $\alpha$ process may be summarized as follows:

(13) The values of $\varepsilon_{10}-\varepsilon_{\infty}$ depended on extension, i.e., these values decreased with increasing extension ratio in the whole temperature range studied, as shown in Figure 10.

(14) Superpositions of $\varepsilon^{\prime \prime} / \varepsilon_{\max }^{\prime \prime}$ along extension ratio were not possible, as shown in Figure 11.
(15) The apparent shift factor $a^{*}$ increased with increasing extension ratio, as shown in Figure 12.

(16) The activation enthalpy $\Delta H^{\neq}$increased with increasing extension ratio, as shown in Figure 14.

It has been confirmed that the superposability of the $\alpha$ process was not affected by the isotropic strain in terms of elevated pressure for some polymers ${ }^{1}$ and the present sample, ${ }^{16}$ i.e., the shape of $\varepsilon^{\prime \prime} / \varepsilon_{\max }^{\prime \prime}$ was independent of pressure. This fact indicates that the elongational strain acts on the $\alpha$ process in a different manner from other thermodynamic variables, e.g., temperature and pressure.

If the function of $\ln a^{*}$ can be considered to be a single-valued function of temperature $T$, pressure $P$, and tensile force $f$ (this assumption has been used in the definition of the activation enthalpy), the activation length $\Delta L^{\neq}$may be defined in the same manner as the activation volume:

$$
\Delta L^{\neq}=R T\left(\frac{\partial \ln a^{*}}{\partial f}\right)_{P T}=R T\left(\frac{\partial \ln a^{*}}{\partial \lambda}\right)_{P T}\left(\frac{\partial f}{\partial \lambda}\right)_{P T}^{-1}
$$

The physical meanings of $\Delta L^{\neq}$is obscure, as is activation volume. However, the value of $\Delta L^{\neq}$ may be a measure of the ease of the relaxation process, and the term $\left(\partial \ln a^{*} / \partial \lambda\right)_{P T}$ may indicate an extension dependence of the mobility of molecular motions. Since the value of $(\partial f / \partial \lambda)_{P T}$ is always positive from the thermodynamic stability condition, the signs of $\Delta L^{\neq}$and $\left(\partial \ln a^{*} / \partial \lambda\right)_{P T}$ coincide in the liquid state. In the glassy state, however, the value of $\ln a^{*}$ can not be regarded as a single-valued function of $T, P$, and $f$, and it is necessary to introduce some new internal variables. ${ }^{17,18}$

From Observation (15), the value of $(\partial \ln a / \partial \lambda)_{P T}$ is positive, and the value of $\Delta L^{\neq}$may also be positive. Therefore the molecules may become more difficult to move with increasing elongational strain. Observation (16) also indicates the difficulty of the molecular motion in the stretched state. The two investigations, by static dielectric constants and by shift factors, are not inconsistent with each other and indicate that the molecular motion with respect to the $\alpha$ 
process would be restricted by elangational strain. The following presumptions may be made in the high temperature region:

(17) It seems that the value of $\left(\partial \log a^{*} / \partial \lambda\right)_{P T}$ becomes zero at about $300^{\circ} \mathrm{K}$ by a linear extrapolation, as shown in Figure 13.

(18) It seems that the magnitude of the extension dependence of $\varepsilon_{10}-\varepsilon_{\infty}$ becames zero at about $450^{\circ} \mathrm{K}$, from the estimate in Figure 10 .

The apparent approach of the nature of the $\alpha$ process to that of the $\beta$ process at higher temperatures was pointed out by Mikhailov, Hartt, Johari, and Williams, et al..$^{419-22}$ In the $\beta$ process, the values of $\left(\partial \log a^{*} / \partial \lambda\right)_{P T}$ and the magnitude of the extension dependences of $\varepsilon_{20}-\varepsilon_{\infty}$ were small. It is interesting to point out that such apparent approach is also indicated in the extension dependences of the shift factors and the static dielectric constants.

The study of sound velocity in chlorinated polyethylene vulcanizate suggested that the situation of the molecular motion along the extension direction in the liquid state became more similar to that in the glassy state with increasing extension ratio. ${ }^{7}$ The differences in the static dielectric constants between the liquid and glass at the glass transition temperature, $\Delta \varepsilon$, are indicated in Table I. That difference decreased slightly with increasing extension ratio, but this decrease was much smaller than that of the difference in sound velocity between the glass and liquid. Therefore it may be said that the situation of the molecular motion pointed out in the sound velocity measurement has not been observed in the dielectric properties which are concerned in the molecular motion perpendicular to the extension direction.

\section{Glass Transition Points}

Figure 16 shows $(\partial T / \partial \lambda)_{P \ln a^{*}}$ as a function of temperature. The $(\partial T / \partial \lambda)_{P} \ln a^{*}$ is defined by

$$
\left(\frac{\partial T}{\partial \lambda}\right)_{P \ln a^{*}}=-\left(\frac{\partial \ln a^{*}}{\partial \lambda}\right)_{P T} /\left(\frac{\partial \ln a^{*}}{\partial T}\right)_{P}
$$

The value of $(\partial T / \partial \lambda)_{P \ln a^{*}}$ means the extension dependence of the temperature at which the system studied has the same mobility. If $(\partial T / \partial \lambda)_{P} \ln a^{*}$ is extrapolated to the dilatometric glass transition temperature, we may obtain the extension dependence of the glass transition temperature

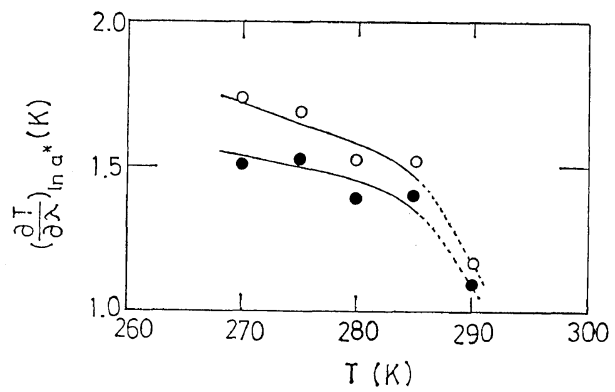

Figure 16. Variation of $(\partial T / \partial \lambda)_{P} \ln a^{*}$ in the $\alpha$ process with temperature at the extension ratios of $\bigcirc, 1.00$ and $O, 1.95$.

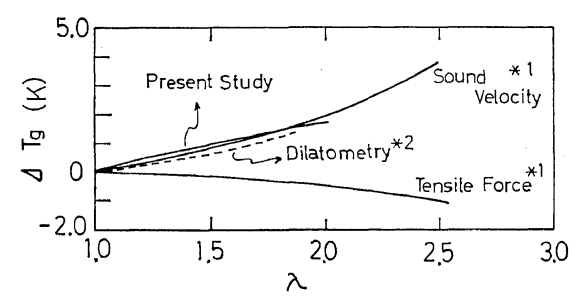

Figure 17. Variation of the change in the glass transition temperature with extension ratio in various methods: $* 1$, obtained by $\mathrm{M}$. Naoki and T. Nose in chlorinated polyethylene vulcanizate; 5 $* 2$, obtained by $\mathrm{A}$. Komatsu and $\mathrm{T}$. Nose in styrene-butadiene copolymer. ${ }^{16}$

observed in the dilatometric time scale. Figure 17 shows the change in the glass transition temperature, $\Delta T_{\mathrm{g}}$, with extension which was obtained by integrating the dependence of the dielectric glass transition points in the dilatometric time scale. The results of the sound velocity and the tensile force measurements for the present sample ${ }^{7}$ and that of the dilatometry for styrenebutadiene copolymer ${ }^{14}$ are also shown in Figure 17. Except for the case of the tensile force, the $\Delta T_{\mathrm{g}}$ values were similar below the extension ratio of about 2. However, the curvature of the $\Delta T_{\mathrm{g}} v s . \lambda$ plot obtained by dielectric measurement was opposite to those from the dilatometric and the sound velocity measurements, i.e., the former had a negative quadratic differential coefficient and the latter had positive ones. To discuss the slope of $\Delta T_{\mathrm{g}} v s . \lambda$ curve, experimental accuracies might be insufficient in all but the dielectric measurements. In the region above the extension ratio of about 2, large values of 
$\Delta T_{\mathrm{g}}$ for some rubbers were presented by Gee, et al. ${ }^{23}$ but new problems such as orientation or crystallization in the region may arise at such high extension ratios.

In Figure 16, the value of $(\partial T / \partial \lambda)_{P} \ln a^{*}$ seems to reach zero at about $310^{\circ} \mathrm{K}$ if we extrapolate the dotted line to higher temperatures. This fact is caused by the decrease in $\Delta L^{\neq}$with increasing temperature. If this extrapolation is appropriate throughout, the following situation may be expected: "When the sample is stretched at a temperature above $310^{\circ} \mathrm{K}$, the glass transition temperature observed in a high frequency measurement will either stay the same or decrease with increasing extension ratio. At that temperature we may be able to observe the glass transition point by that frequency measurement."

The extension dependence of the freezing temperature $T_{\mathrm{g} \beta}$ of the $\beta$ process may be given by the same manner as in the $\alpha$ process. It is supposed that the static freezing of the $\beta$ process arises near the apparent extinction temperature $T_{0}$, which is indicated in Figure 5. Both values of $\left(\partial \ln a^{*} / \partial T\right)_{P}$ and $\left(\partial \ln a^{*} / \partial \lambda\right)_{P T}$ are negative in the low temperature region as shown in Figure 7, and consequently, it is found from eq (5-1) that the value of $(\partial T / \partial \lambda)_{P \ln a^{*}}$ is negative. These estimations are not appropriate for the $\beta$ process as discussed above, but it may be supposed that the extension dependence of $T_{\mathrm{g} \beta}$ is negative, i.e., the freezing temperature of the $\beta$ process might be lowered by increasing the extension ratio. On the other hand, the extension dependence of $T_{0}$ is also negative, as shown in Figure 5 . It is interesting to point out that these two facts, estimated in different manners, (i.e., one was obtained from the consideration of the molecular mobility and the other from the behavior of the static dielectric constants,) are not inconsistent with each other and may indicate that the freezing temperature of the $\beta$ process, even if this freezing state is merely hypothetical, might be lowered by increasing the elongational strain.

\section{CONCLUSION}

The static dielectric constants as a function of temperature and elongational strain were obtained in the vicinity of the glass transition region for chlorinated polyethylene valcanizate. It was found that the static dielectric constants increased linearly with an increase in temperature in the glassy state and linearly with an increase in reciprocal temperature in the liquid state. The extension dependence of the static dielectric constants was negative in the vicinity of the glass transition region, in both the glassy and liquid state. However this dependence seemed to vanish in the regions far above and below the glass transition temperature.

The mobility of the molecular motions suggested from the apparent shift factors decreased with an increase in elongational strain in the $\alpha$ process, whereas it changed very little or seemed to increase slightly in the $\beta$ process.

The superposability along temperature was confirmed both in the $\alpha$ and $\beta$ process. However, the superposability along extension ratio was impossible in the $\alpha$ process and was not confirmed in the $\beta$ process because of experimental errors. Therefore, the role of elongational strain in the mobility of molecular motions was considered to be different from those of pressure and temperature in the $\alpha$ process.

In the high temperature region, an apparent approach of the $\alpha$ process to the $\beta$ process was found in some observations, i.e., in the extension dependence of the static dielectric constants and the apparent shift factors.

The change in the dielectric glass transition temperature for the dilatometric time scale with elongational strain was obtained. It was found that the glass transition temperatures in the dielectric measurement decreased with an increase in elongational strain similar to the results of the sound velocity and the dilatometric measurements, but not to those of the tensile force measurement. This extension dependence of the dielectric glass transition temperature seemed to vanish at high temperatures.

The apparent extinction point of the $\beta$ process was observed. It was found that both of the apparent extinction temperature and the apparent freezing temperature of the $\beta$ process would decrease with an increase in elongational strain. This behavior was different from that of the $\alpha$ 
process.

Acknowledgments. Particular acknowledgments are due to Mr. M. Motomura who designed the apparatus studied, to Dr. T. Saito at the Research Laboratories of Showa-Denko Company who prepared the sample studied, and to $\mathrm{Mr}$. $\mathrm{H}$. Nakauchi at the Research Laboratories of Bridgestone Company who carried out the vulcanization of this sample.

\section{REFERENCES}

1. G. Williams, Trans. Faraday Soc., 60, 1548 (1964); ibid., 60, 1556 (1964); ibid., 61, 1564 (1965); G. Williams, D. C. Watts, and J.P. Nottin, ibid., 68, 16 (1972).

2. S. Saito, H. Sasabe, T. Nakajima, and K. Yada, J. Polym. Sci., Part A-2, 6, 1297 (1968); H. Sasabe and T. Saito, ibid., Part A-2, 6, 1401 (1968).

3. J. Koppolman, Electrochemie, 65, 689 (1961).

4. G. Williams and D. C. Watts, Trans. Faraday Soc., 67, 1971 (1971); ibid., 68, 2793 (1972).

5. H. Sasabe and T. Saito, J. Polym. Sci., Part A-2, 6, 1401 (1968).

6. P. Mason, J. Appl. Polym. Sci., 1, 63 (1959); P. Mason, Soc. Chem. Inds., Monograph No. 5, London, 1461 (1959).

7. M. Naoki and T. Nose, Polymer J., 6, 45 (1974).

8. T. Saito, K. Yamaguchi, and S. Hayashi, Kobunshi Kagaku (Chem. High Polymers), 29,
78 (1972).

9. T. Saito, Ph. D. Thesis presented at the Department of Polymer Chemistry, Tokyo Institute of Technology, Tokyo, in September, 1972.

10. T. Saito, Y. Matsumura, and S. Hayashi, Polymer J., 1, 639 (1970).

11. S. Osaki, S. Uemura, and Y. Ishida, J. Polym. Sci., Part A-2, 9, 585 (1971).

12. R. G. Christensen and C. A. J. Hoeve, J. Polym. Sci., Part A-1, 8, 1053 (1970).

13. G. Allen, M. J. Kirkham, J. Padget, and C. Price, Trans. Faraday Soc., 67, 1278 (1971).

14. A. Komatsu and T. Nose, Rept. Progr. Polym. Phys. Japan, 14, 309 (1971).

15. J.D. Ferry, M. L. Williams, and E. R. Fitzgerald, J. Phys. Chem., 59, 403 (1955).

16. M. Naoki, M. Motomura, and T. Nose, to be published.

17. R. O. Davies and G. O. Jones, Proc. Roy. Soc., Ser. $A$, 217, 26 (1953).

18. T. Nose, Polymer J., 4, 217 (1973); K. Tanaka, T. Nose, and T. Hata, Rept. Progr. Polym. Phys. Japan, 15, 229 (1972).

19. G. P. Mikhailov and A. M. Labanov, Soviet Phys. Solid State, 5, 1339 (1964).

20. J. K. Hartt and E. F. Carr, J. Chem. Phys., 36, 2523 (1962).

21. M. Goldstein, ibid., 51, 3728 (1969).

22. G. P. Johari and M. Goldstein, ibid., 53, 2372 (1970).

23. G. Gee, P. N. Hartley, J. B. M. Herbert, and H. A. Lanceley, Polymer, 1, 365 (1960). 